BA-99-05

FERMILAB-Pub-99/007-T

January 1999

\title{
Predicting quark and lepton masses and mixings
}

\author{
Carl H. Albright ${ }^{1}$ \\ Department of Physics \\ Northern Illinois University, DeKalb, IL 60115 \\ and Fermi National Accelerator Laboratory \\ P.O. Box 500, Batavia, IL 60510 \\ S.M. Barr ${ }^{2}$ \\ Bartol Research Institute \\ University of Delaware \\ Newark, DE 19716
}

\begin{abstract}
A model is presented that fits the quark and lepton masses and mixings wherein five dimensionless parameters and a phase account for fifteen dimensionless observables. Among these are the Wolfenstein parameters $\rho$ and $\eta$, the $\nu_{e}-\nu_{\mu}$ and $\nu_{e}-\nu_{\tau}$ mixing angles which are predicted to be small and comparable while the $\nu_{\mu}-\nu_{\tau}$ mixing angle is predicted to be large. The model is based on supersymmetric $S O(10)$ with the form of the mass matrices motivated by simplicity at the level of grand unification.
\end{abstract}

PACS numbers: 12.15.Ff, 12.10.Dm, 12.60.Jv, 14.60.Pq

1E-mail: albright@fnal.gov; ${ }^{2}$ E-mail: smbarr@bartol.udel.edu 
In an earlier paper with Babu [1], the authors have proposed a model based on supersymmetric $S O(10)$ for the masses and mixings of the heavier two families of quarks and leptons. One of the guiding principles in constructing the model was that the mass terms should be of a kind that can arise simply in $S O(10)$ with small representations of Higgs fields [2]. Two critical points emerged from that work: namely, that it is quite natural in unified theories for there to be flavor-asymmetric contributions to the fermion mass matrices, and that these can explain some of the puzzling features of the spectrum. In particular, it was shown that a flavor-asymmetric term can account in a simple way for the apparent fact that the $\nu_{\mu}-\nu_{\tau}$ mixing is very large compared to $V_{c b}$.

The present work is an extension of that earlier model to include the first family. We find that it is only necessary to introduce two new parameters, one of which has a complex phase, in order to account for seven quantities that involve the first family. In doing so, several interesting predictions result, including the values of the $\nu_{e}-\nu_{\mu}$ and $\nu_{e}-\nu_{\tau}$ mixing angles, and a relationship between the real and imaginary parts of $V_{u b}$, i.e., between the Wolfenstein parameters $\rho$ and $\eta$. Altogether, as will be seen, the model leads to nine predictions.

\section{The mass matrices.}

The mass matrices proposed have the following forms:

$$
\begin{array}{rlrl}
U^{0} & =\left(\begin{array}{ccc}
0 & 0 & 0 \\
0 & 0 & \epsilon / 3 \\
0 & -\epsilon / 3 & 1
\end{array}\right) M_{U}, & D^{0}=\left(\begin{array}{ccc}
0 & \delta & \delta^{\prime} \\
\delta & 0 & \sigma+\epsilon / 3 \\
\delta^{\prime} & -\epsilon / 3 & 1
\end{array}\right) M_{D} \\
N^{0} & =\left(\begin{array}{ccc}
0 & 0 & 0 \\
0 & 0 & -\epsilon \\
0 & \epsilon & 1
\end{array}\right) M_{U}, & L^{0} & =\left(\begin{array}{ccc}
0 & \delta & \delta^{\prime} \\
\delta & 0 & -\epsilon \\
\delta^{\prime} & \sigma+\epsilon & 1
\end{array}\right) M_{D}
\end{array}
$$

where $U, D, L$, and $N$ stand, respectively, for the up-quark, down-quark, charged-lepton, and the neutrino Dirac mass matrices. The zero superscripts stand here and throughout for quantities defined at the grand unification (GUT) scale. The convention we use is that the left-handed fermions multiply these matrices from the right, while the right-handed fermions multiply them from the left. (Thus, it is the entries below the main diagonal that control the quark and lepton mixings that are observed.) 
It is immediately apparent that the matrix $U^{0}$ is rank-2, corresponding to a massless $u$ quark. We imagine that some small higher-order effects give $u$ a mass of several $\mathrm{MeV}$. The fact that $m_{u}$ must arise at higher order than $m_{d}$ and $m_{e}$ is not a flaw but a qualitative success of the model. The point is that a several-MeV $u$-quark mass corresponds to $m_{u}^{0} / m_{t}^{0} \approx 10^{-5}$. This is very much smaller than the corresponding ratios $m_{d}^{0} / m_{b}^{0} \approx 10^{-3}$ and $m_{e}^{0} / m_{\tau}^{0} \approx 0.3 \times 10^{-3}$, and so it is reasonable to suppose that it is a higherorder effect.

By field redefinitions one can make all the elements of the matrices in Eq. (1) real except for $\delta^{\prime}$. (There can also be a relative phase initially between $\sigma$ and $\epsilon$, which was called $\alpha$ in [1]. After phase redefinitions, however, this parameter survives only in the entries $D_{23}^{0}$ and $L_{32}^{0}$, which can be approximated by $\sigma+\frac{\epsilon}{3} \cos \alpha$ and $\sigma+\epsilon \cos \alpha$, respectively, since as will be seen later $\epsilon / \sigma$ is a small parameter. As $\cos \alpha$ only appears in terms subleading in $\epsilon / \sigma$, it has only a few-percent effect on predictions. In [1] it was found that the fits prefer $\alpha=0$, so we will assume that value henceforth in this paper.) Effectively, then, the mass matrices in Eq. (1) contain five real dimensionless parameters and one phase: $\left(M_{U} / M_{D}\right), \sigma, \epsilon, \delta,\left|\delta^{\prime}\right|$, and $\arg \delta^{\prime}$.

We will first describe the kinds of $S O(10)$ operators and diagrams that give rise to the entries shown in (1), and explain the rationale for the those entries. Then we shall proceed to discuss the fit to the data and the predictions of the model.

\section{The origin and rationale of the matrices.}

The (33) entries of the mass matrices contribute to the masses of the heaviest family of quarks and leptons. As is true of most grand unified models of fermion masses, these entries can come from the minimal kind of Yukawa term. Since ours is an $S O(10)$ model, these entries arise simply from the operator $\left(\mathbf{1 6}_{3} \mathbf{1 6}_{3}\right) \mathbf{1 0}_{H}$. Here and throughout, the subscript ' $H$ ' stands for a Higgs multiplet, and a numerical subscript stands for the family number of a quark/lepton multiplet. The parameters $M_{U}$ and $M_{D}$ in Eq. (1) come from the $S U(2)_{L} \times U(1)_{Y}$-breaking vacuum expectation values $(\mathrm{VEVs})$ in $\mathbf{5}\left(\mathbf{1 0}_{H}\right)$ and $\overline{\mathbf{5}}\left(\mathbf{1 0}_{H}\right)$, respectively. We use $(\mathbf{p}(\mathbf{q})$ to represent a $\mathbf{p}$ multiplet of $S U(5)$ contained in a $\mathbf{q}$ multiplet of $S O(10)$.

While the masses of the third family satisfy the minimal $S U(5)$ (and $S O(10))$ relation $m_{b}^{0} \cong m_{\tau}^{0}$, the middle family does not satisfy the corre-

sponding $m_{s}^{0} \cong m_{\mu}^{0}$ relation. As is well known, however, a good fit is given 
by the Georgi-Jarlskog relation [3], $m_{s}^{0} \cong m_{\mu}^{0} / 3$. Perhaps the simplest way to get a factor of $1 / 3$ into the elements of the mass matrices is through an adjoint Higgs multiplet, $\mathbf{4 5} \mathbf{5}_{H}$. Such a Higgs multiplet is needed in any case for the breaking of $S O(10)$ down to the standard model group. In the simplest breaking scheme that naturally solves the "doublet-triplet splitting problem", the so-called Dimopoulos-Wilczek or "missing VEV" mechanism [⿴囗十, there is an adjoint Higgs multiplet whose VEV is proportional to the $S O(10)$ generator $B-L$. This is in fact just what is needed to get a relative factor of $1 / 3$ between entries in the quark and lepton mass matrices.

The lowest-dimension Yukawa operators that contains the adjoint Higgs multiplet are products of the form $\mathbf{1 6}_{i} \mathbf{1 6} \mathbf{1 0}_{j} \mathbf{4} \mathbf{5}_{H}$. There are two ways to contract the $S O(10)$ indices, but it is easy to show that if $\left\langle\mathbf{4} \mathbf{5}_{H}\right\rangle \propto B-L$, then only the flavor-antisymmetric piece contributes to the mass matrices. This accounts for the form of the " $\epsilon$ " entries in Eq. (1). Note that these appear with a factor of $1 / 3$ in the quark matrices and 1 in the lepton matrices, reflecting the generator $B-L$. One also sees that because the operator being discussed contains the same $\mathbf{1 0}_{H}$ that appears in the Yukawa operator for the (33) entries, the $\epsilon$ entries of $U^{0}$ and $N^{0}$ are $M_{U} / M_{D}$ times those of $D^{0}$ and $L^{0}$.

The operator $1 \mathbf{6}_{2} \mathbf{1 6}_{3} \mathbf{1 0} \mathbf{4 5}_{H}$ needed for the $\epsilon$ entries arises in a simple fashion by integrating out a superheavy (i.e. GUT-scale) family plus conjugate-family pair: $\mathbf{1 6}+\overline{\mathbf{1 6}}$ and is naturally of order $1 / M_{G}$ as required. This is shown in Fig. 1. Such an operator could also arise from Planck-scale physics, but it would then be too small.

The next pieces of the mass matrices to be discussed are the " $\sigma$ " entries. These have several rationales, and are in fact the key to the whole model. We shall first explain how such entries arise, and then why they are important for getting a realistic model.

The feature of the $\sigma$ entries that distinguishes them from what is found in most published models is that they are neither flavor-symmetric nor flavorantisymmetric, but rather "lopsided": $\sigma$ appears in $D_{23}^{0}$ but not in $D_{32}^{0}$. Though it is not common to consider such lopsided forms, there is really no reason from the point of view of grand unification not to do so. In fact, it may well be the neglect of such possibilities that has handicapped the search for realistic quark and lepton mass matrices.

The $\sigma$ entries arise very simply from integrating out an $S O(10)$ vector 10 of quarks and leptons as shown in Fig. 2. The resulting operator is of the form $\left[\mathbf{1 6}_{2} \mathbf{1 6}_{H}\right]_{10}\left[\mathbf{1 6}_{3} \mathbf{1 6}_{H}^{\prime}\right]_{10}$. The notation $[\ldots]_{10}$ indicates that what is 
contained in the brackets is contracted into a $\mathbf{1 0}$ of $S O(10)$ as a result of the virtual 10 fermions appearing in Fig. 2. The Higgs multiplet denoted $\mathbf{1 6}_{H}$ is assumed to develop a GUT-scale VEV in the $\mathbf{1}(\mathbf{1 6})$ direction, while the other spinor Higgs, denoted $\mathbf{1 6}_{H}^{\prime}$, gets a weak-scale $S U(2)_{L}$-doublet VEV in the $\overline{\mathbf{5}}(\mathbf{1 6})$ direction. Thus, in $S U(5)$ notation, the operator from Fig. 2 gives $\left(\overline{\mathbf{5}}_{2} \mathbf{1 0}_{3}\right)\left\langle\overline{\mathbf{5}}_{H}^{\prime}\right\rangle\left\langle\mathbf{1}_{\mathbf{H}}\right\rangle$.

One observes that since the left-handed down-quarks are in 10's of $S U(5)$, not $\overline{5}$ 's, this operator contributes to $D_{23}$ and not $D_{32}$. On the other hand, since the left-handed leptons are in $\overline{5}$ 's, not 10's, the operator contributes to $L_{32}$ and not $L_{23}$. As we shall see, this fact that the $\sigma$ entry is transposed in $L$ relative to that in $D$ is very important for understanding the large $\nu_{\mu}-\nu_{\tau}$ mixing that may have been seen in the atmospheric neutrino data. This transposition is a consequence of the group theory of $S U(5)$ which also implies that $D=L^{T}$ in the minimal $S U(5)$ model.

The upshot is that integrating out 10's of fermions in $S O(10)$ can quite typically give just such lopsided entries as the $\sigma$ entries in Eq. (1). Note that the $\sigma$ entries only appear in $D$ and $L$, and not in $U$ and $N$, following from the fact that $\mathbf{1 6}_{H}^{\prime}$ contains only $\overline{\mathbf{5}}$ and not $\mathbf{5}$ of $S U(5)$.

Now, why should such lopsided entries help to get a realistic model? It turns out that these $\sigma$ entries play at least six vital roles:

(i) In the first place, it is easy to see from the seesaw-type formulas, that setting $\sigma=0$ in Eq. (1) would give $m_{s}^{0} / m_{b}^{0} \cong(\epsilon / 3)^{2}$ and $m_{\mu}^{0} / m_{\tau}^{0} \cong \epsilon^{2}$. Thus one would get a ratio of ratios of $1 / 9$ instead of the desired Georgi-Jarlskog value of $1 / 3$. If, however, $\sigma$ is present and is large compared to $\epsilon$, then one has $m_{s}^{0} / m_{b}^{0} \sim \sigma \epsilon / 3$, and $m_{\mu}^{0} / m_{\tau}^{0} \sim \sigma \epsilon$, so that the desired Georgi-Jarlskog factor emerges.

(ii) Without the $\sigma$ entries, the (23) blocks of $D$ and $U$ would be proportional, and consequently there would be no KM mixing for those families, that is $V_{c b}$ would vanish.

(iii) Moreover, the lopsided $\sigma$ entries explain a peculiar fact about the magnitude of $V_{c b}$. If $D_{23}$ and $D_{32}$ were of equal magnitude, as is the case with the purely flavor-symmetric or antisymmetric forms usually assumed, then $V_{c b}$ would get a contribution from $D$ that is $\sqrt{m_{s} / m_{b}}$, which is much too big (by about a factor of 4). By having the large lopsided contribution with $\sigma>\epsilon$, one finds $D_{32} \ll D_{23}$, which leads to $V_{c b} \ll \sqrt{m_{s} / m_{b}}$, as observed. This cures one of the main problems with textures of the Fritzsch type [5] as noted previously by Branco and Silva-Marcos [6] in a phenomenological 
study of the quark sector. Other authors [7] have also recently appreciated this fact in the context of $S U(5)$ models.

(iv) Because $\sigma$ naturally only appears in $D$ and $L$ but not in $U$, one explains why $m_{c} / m_{t} \ll m_{s} / m_{b}$ and $m_{\mu} / m_{\tau}$, as actually observed. In the absence of the $\sigma$ entries, one would obtain the minimal $S O(10)$ prediction $m_{c}^{0} / m_{t}^{0}=m_{s}^{0} / m_{b}^{0}$, which is wrong by about a factor of 20 .

(v) The fact that the large $\sigma$ entry appears transposed in $L$ relative to $D$ means that it makes the (32) mixing of the left-handed leptons large compared to the corresponding mixing of the left-handed quarks. One thus explains why $\nu_{\mu}-\nu_{\tau}$ mixing is much larger than $V_{c b}$, which is one of the great puzzles in understanding the atmospheric neutrino oscillations.

(vi) Finally, we shall see later that the $\sigma$ entries explain why the $\nu_{e}-\nu_{\mu}$ mixing angle is significantly smaller than $\sqrt{m_{e} / m_{\mu}}$, assuming the small-angle MSW solution of the solar neutrino problem.

What remains to be explained about the mass matrices in Eq. (1) are the entries $\delta$ and $\delta^{\prime}$. These arise very simply from the diagrams in Fig. 3. Note that because the effective operators are again of the form $\mathbf{1 6}_{i} \mathbf{1 6}_{j} \mathbf{1 6}_{H}^{\prime} \mathbf{1 6}_{H}$, as for the $\sigma$ entries, these diagrams only contribute to $D$ and $L$, and not to $U$ and $N$. Note also that because they come from integrating out a $\mathbf{1 0}$ of Higgs, they are flavor-symmetric. These contributions are assumed to be much smaller than the others.

The fact that the above terms appear and no others presupposes that some Abelian symmetries exist that distinguish among families. Such symmetries have been used by many authors. For an interesting recent publication with a number of references cited, see [8]. We note that realistic models involving such symmetries can be constructed giving rise to the matrices in Eq. (2). Much of the structure of such a model has already been described in [1] and [2]. In addition to what was presented there, there have to be $S O(10)$ 10's of Higgs fields to be integrated out to give the $\delta$ and $\delta^{\prime}$ entries, as in Fig. 3. Such fields and the necessary couplings can be introduced, with appropriate Abelian symmetry, without destabilizing the gauge hierarchy or creating other difficulties [9].

\section{The predictions of the matrices.}

In expressing the predictions of the model, it proves convenient to use instead of the parameters $\delta$ and $\delta^{\prime}$, the linear combinations $t_{R} \equiv \delta \sqrt{\sigma^{2}+1} /(\sigma \epsilon / 3)$ and $t_{L} \equiv\left(\delta-\sigma \delta^{\prime}\right) /(\sigma \epsilon / 3)$. Essentially, $t_{L}$ is the Cabibbo angle, and $t_{R}$ is 
the corresponding mixing angle of the right-handed $d$ and $s$ quarks. That is to say, if one diagonalizes the (23) block of $D^{0}$ in Eq. (1), and calls the resulting matrix $D^{0^{\prime}}$, then $t_{L} \cong D_{21}^{0^{\prime}} / D_{22}^{0^{\prime}}$ and $t_{R} \cong D_{12}^{0^{\prime}} / D_{22}^{0^{\prime}}$. In the phase convention being adopted, $t_{R}$ is real and $t_{L}$ has a phase that shall be called $\theta$.

The parameters, then, are $M_{U} / M_{D}, \sigma, \epsilon, t_{R},\left|t_{L}\right|$, and $\theta$. In terms of these one has the following relations, which are given only to leading order in the small parameters $\epsilon, t_{R}$, and $t_{L}$ :

$$
\begin{aligned}
& m_{t}^{0} / m_{b}^{0} \cong M_{U} / M_{D}, \\
& m_{\tau}^{0} / m_{b}^{0} \cong 1 \\
& m_{c}^{0} / m_{t}^{0} \cong \frac{1}{9} \epsilon^{2} \\
& m_{s}^{0} / m_{b}^{0} \cong \frac{1}{3} \epsilon \frac{\sigma}{\sigma^{2}+1} \\
& m_{\mu}^{0} / m_{\tau}^{0} \cong \epsilon \frac{\sigma}{\sigma^{2}+1} \\
& m_{u}^{0} / m_{c}^{0} \cong 0 \\
& m_{d}^{0} / m_{s}^{0} \cong\left|t_{L}\right| t_{R} \\
& m_{e}^{0} / m_{\mu}^{0} \cong \frac{1}{9}\left|t_{L}\right| t_{R} \\
& V_{c b}^{0} \cong \frac{1}{3} \epsilon \frac{\sigma^{2}}{\sigma^{2}+1} \\
& V_{u s}^{0} \cong\left|t_{L}\right| \\
& V_{u b}^{0} \cong \frac{1}{3} \epsilon \frac{1}{\sigma^{2}+1}\left[\sqrt{\sigma^{2}+1} t_{R} e^{-i \theta}-\left|t_{L}\right|\right] \\
& U_{\mu 3} \equiv \sin \theta_{\mu \tau} \cong-\frac{\sigma}{\sqrt{\sigma^{2}+1}}+O(\epsilon) \\
& U_{e 2} \cong-\frac{1}{3} t_{R} \cos \theta_{\mu \tau} \\
& U_{e 3} \cong \frac{1}{3} t_{R} \sin \theta_{\mu \tau}
\end{aligned}
$$

Predictions involving only the second and third families were given already 
in [1], where a fit was done using more exact expressions, and taking into account renormalization-group running from the unification scale down to low scales as well as radiative corrections to the heavy quark masses coming from supersymmetry. From $m_{\mu} / m_{\tau}$ and $V_{c b}$ which have small experimental uncertainties, $\epsilon$ and $\sigma$, which was previously called $\rho$ in [1], were extracted; cf., Eqs. (2e) and (2i). It was found that the best fit was given by

$$
\begin{aligned}
& \epsilon \cong 0.139, \\
& \sigma \cong 1.81 .
\end{aligned}
$$

From Eq. (2c) one can then obtain a prediction for the charm quark mass. In [1] it was found that $m_{c}\left(m_{c}\right)=1.10 \pm 0.1 \mathrm{GeV}$, in satisfying agreement with the experimental value $1.27 \pm 0.1 \mathrm{GeV}$.

From Eq. (2l) one sees that the model predicts a large, and in fact a nearly maximal, mixing of $\nu_{\mu}$ and $\nu_{\tau}$. The element $\left|U_{\mu 3}\right|$, which describes this mixing, receives a large contribution from diagonalizing the mass matrix of the charged leptons, $L$. This large contribution is well determined in the model, since $L$ is known, and comes out to be approximately $\sigma / \sqrt{\sigma^{2}+1} \cong$ 0.87 . However, $\left|U_{\mu 3}\right|$ also receives a small contribution from diagonalizing the neutrino mass matrix, which has the usual seesaw form $M_{\nu}=-N^{T} M_{R}^{-1} N$ [10]. While $N$ is known in the model, $M_{R}$ is not - at least not without some further assumptions. Nevertheless, one can say that the contribution to $\left|U_{\mu 3}\right|$ from diagonalizing $M_{\nu}$ is of order $\epsilon$, following from the form of $N$. Though relatively small, this contribution makes impossible a precise prediction of the $\nu_{\mu}-\nu_{\tau}$ mixing angle, though it is possible to say that it is nearly maximal. This source of uncertainty does not affect the predictions for the other two lepton mixing angles, however, because of the fact that $N$, and therefore $M_{\nu}$, have zeros in the first row and column. These zeros are doubtless lifted by the higher order effects that generate $m_{u}$, but it is easy to see that this should have a negligible effect on $U_{e 2}$ and $U_{e 3}$.

The other predictions for the heavy families are that $m_{b}^{0} \cong m_{\tau}^{0}$ from Eq. (2b) and $m_{s}^{0} / m_{b}^{0} \cong \frac{1}{3} m_{\mu}^{0} / m_{\tau}^{0}$ from Eqs. (2d) and (2e). Both of these are well-known relations, and work well in the context of supersymmetric grand unification. The foregoing four predictions were thoroughly discussed in [1].

Let us turn now to the eight quantities that involve the first family: $m_{e}$, $m_{d}, m_{u}, U_{e 2}, U_{e 3}, V_{u s}, \operatorname{Re}\left(V_{u b}\right)$, and $\operatorname{Im}\left(V_{u b}\right)$. These depend on the three additional parameters $t_{R},\left|t_{L}\right|$, and $\theta$. The approximate magnitudes of $t_{R}$ 
and $\left|t_{L}\right|$ can be estimated in a simple way, as follows. Comparing the wellknown empirical relation $\sin \theta_{c} \cong \sqrt{m_{d} / m_{s}}$ with Eqs. $(2 \mathrm{j})$ and $(2 \mathrm{~g})$, one sees immediately that $\left|t_{L}\right| \simeq t_{R} \simeq \sin \theta_{c}$. This enables one to read off an estimate of the Wolfenstein parameters [11] $\rho$ and $\eta$ from Eq. 2. To leading order in the Wolfenstein parameter $\lambda$, one has that $\rho+i \eta=\left(V_{u b} V_{c s} / V_{c b} V_{u s}\right)^{*}$. But the value of that invariant is given in this model (to leading order in $\epsilon, t_{R}$, and $\left.t_{L}\right)$ by

$$
\left(\frac{V_{u b} V_{c s}}{V_{c b} V_{u s}}\right)^{*} \cong \frac{1}{\sigma^{2}}\left[\sqrt{\sigma^{2}+1} \frac{t_{R}}{\left|t_{L}\right|} e^{i \theta}-1\right] .
$$

See Eqs. $(2 \mathrm{i}),(2 \mathrm{j})$, and $(2 \mathrm{k})$. Since $\left|t_{L}\right| \simeq t_{R}$ and $\sigma \simeq \sqrt{3}$ one has, roughly, that $\rho+i \eta \simeq \frac{2}{3} e^{i \theta}-\frac{1}{3}$ corresponding to a circle in the $\rho-\eta$ plane.

To get the predictions more exactly, it is best to use $V_{u s}$ and $m_{e} / m_{\mu}$, which have the smallest experimental uncertainties, to determine $t_{R}$ and $\left|t_{L}\right|$; cf., Eqs. (2h) and (2j). Employing more exact expressions than given in Eqs. (2), one finds that

$$
\begin{aligned}
& \left|t_{L}\right| \cong 0.239 \\
& t_{R} \cong 0.185 .
\end{aligned}
$$

The phase of $t_{L}$, denoted $\theta$, is so far undetermined. Substituting these values into Eq. (4), and taking into account the corrections to that equation that are higher order in $\epsilon, t_{R}$, and $t_{L}$, one finds

$$
\rho+i \eta \cong 0.47 e^{i \theta}-0.29 .
$$

This circle, plotted as the bold dashed arc in Fig. 4, slices nicely through the presently allowed region [12].

The prediction for the $\nu_{e}-\nu_{\mu}$ mixing, cf. Eq. $2(\mathrm{~m})$, is that

$$
\sin ^{2} 2 \theta_{e \mu} \equiv 4\left|U_{e 2}\right|^{2}\left(1-\left|U_{e 2}\right|^{2}\right) \cong 6.6 \times 10^{-3} .
$$

This is well within the present limits $3 \times 10^{-3}<\sin ^{2} 2 \theta_{e \mu}<8 \times 10^{-3}$ for the small angle MSW solution to solar neutrino problem. The crucial role of the parameter $\sigma$ should be noted. In its absence one would find the familiar type of prediction, $U_{e 2} \simeq \sqrt{m_{e} / m_{\mu}}$, which is much too large. However, since there is a large intermingling of the second and third families caused by the 
parameter $\sigma$ - the intermingling that causes the large $\nu_{\mu}-\nu_{\tau}$ mixing - the angles $U_{e 2}$ and $U_{e 3}$ get intermingled as well. This leads to the suppression of $U_{e 2}$ by a factor of $\cos \theta_{\mu \tau} \simeq 0.7$, which brings it into the experimentally allowed range [12].

This same intermingling gives rise to a distinctive prediction of this model, namely that

$$
\left|U_{e 3}\right| \cong \tan \theta_{\mu \tau}\left|U_{e 2}\right|
$$

Using more exact expressions, one finds that

$$
\sin ^{2} 2 \theta_{e \tau} \cong 1.1 \tan ^{2} \theta_{\mu \tau} \sin ^{2} 2 \theta_{e \mu} .
$$

The remaining two predictions of the model are that $m_{u} \approx 0$, Eq. (2f), which has been discussed already, and that $m_{d}^{0} / m_{s}^{0} \cong 9 m_{e}^{0} / m_{\mu^{0}}$, Eqs. $(2 \mathrm{~g})$ and $(2 \mathrm{~h})$, the other Georgi-Jarlskog relation, which gives $m_{d} / m_{s} \cong 1 / 23$ in excellent agreement with the current-algebra result.

A comment is in order about the robustness of these predictions. In obtaining the value of $t_{R}$, we used $m_{e} / m_{\mu}$ on the grounds that the experimental uncertainty in this quantity is negligible. However, one might question whether Eq. (2h) is trustworthy. The point is that since some higher order effects are presumed to generate a mass for the $u$ quark, the same effects are likely to make a small contribution to the masses of $d$ and $e$ as well, though that is not necessarily the case. For example, $m_{u}$ could arise from $U_{11}^{0} \sim 10^{-5} M_{U}$, and a comparable entry in $L$ of order $L_{11}^{0} \sim 10^{-5} M_{D}$ would affect $m_{e}$ at the $3 \%$ level. This would change $t_{R}$, and thus $U_{e 2}$, by the same amount. The effect of such uncertainties on the prediction for the Wolfenstein parameters $(\rho, \eta)$ is relatively small. The prediction in Eq. (9) is even more solid, since $t_{R}$ cancels out in the ratio of $U_{e 3}$ to $U_{e 2}$.

\section{Conclusions}

We have presented a model of quark and lepton masses and mixings that is based on supersymmetric $S O(10)$. The mass matrices arise from five Yukawa terms, each of which is of a type that arises in a straightforward way from simple tree-level diagrams. The model contains very few parameters, so few that there are nine predictions.

Four of the predictions follow from the basic structure of the model and simple group-theoretic considerations. These are $m_{u} \approx 0$ (which corresponds to the observed fact that $m_{u} / m_{t} \sim 10^{-5}$, much less than $m_{d} / m_{b} \cong 10^{-3}$ 
and $\left.m_{e} / m_{\tau} \cong 0.3 \times 10^{-3}\right) ; m_{b}^{0} \cong m_{\tau}^{0}$ (the well-known and successful $S U(5)$ relation); $m_{s}^{0} / m_{b}^{0} \cong \frac{1}{3} m_{\mu}^{0} / m_{\tau}^{0}$ (one of the Georgi-Jarlskog relations); and $m_{d}^{0} / m_{s}^{0} \cong 9 m_{e}^{0} / m_{\mu}^{0}$ (the other Georgi-Jarlskog relation).

Four other predictions are non-trivial quantitative successes of the model. They are a prediction of the charm quark mass that is correct within the present uncertainties; the prediction of very large mixing between $\nu_{\mu}$ and $\nu_{\tau}$, consistent with the recent discovery of atmospheric neutrino oscillations; a prediction for $V_{u b}$ that slices neatly through the middle of the allowed region for the Wolfenstein parameters $\rho$ and $\eta$; and a prediction for $\sin ^{2} 2 \theta_{e \mu}$ that is well inside the present allowed range for the small-angle MSW solution of the solar neutrino problem.

The last prediction is of a presently unmeasured quantity: $\sin ^{2} 2 \theta_{e \tau} \cong$ $1.1 \sin ^{2} 2 \theta_{e \mu}$.

The model is eminently testable. The most dramatic test would be a measurement of $\theta_{e \tau}$, which could come from improved proton accelerator bounds or perhaps from a muon collider. The next most important and fairly clean tests would come from a resolution of the solar neutrino problem and the consequent precise determination of $\theta_{e \mu}$; and from a measurement of the Wolfenstein parameters $\rho$ and $\eta$. Much more difficult, but hopefully achievable someday, would be precision tests of the predictions for $m_{b}$ and $m_{c}$. This would require considerable knowledge of the spectrum of the sparticles, knowledge of $\tan \beta$ and the Higgs masses, and better determinations of $\alpha_{s}, m_{t}$, and $V_{c b}$ [1].

One of us (CHA) thanks the Fermilab Theoretical Physics Department for its kind hospitality where much of his work was carried out. The research of SMB was supported in part by the Department of Energy under contract No. DE-FG02-91ER-40626. Fermilab is operated by Universities Research Association Inc. under contract No. DE-AC02-76CH03000 with the Department of Energy. 


\section{References}

[1] C.H. Albright, K.S. Babu, and S.M. Barr, Phys. Rev. Lett. 81 (1998) 1167, hep-ph/9802314; to be published in Proceedings of the NEUTRINO 98 Conference, hep-ph/9805266.

[2] C.H. Albright and S.M. Barr, Phys. Rev. D 58 (1998) 013002, hepph/9712488.

[3] H. Georgi and C. Jarlskog, Phys. Lett. 86B (1979) 297.

[4] S. Dimopoulos and F. Wilczek, report No. NSF-ITP-82-07 (1981), in The unity of fundamental interactions, Proceedings of the 19th Course of the International School of Subnuclear Physics, Erice, Italy, 1981, ed. A. Zichichi (Plenum Press, New York, 1983); K.S. Babu and S.M. Barr, Phys. Rev. D 48 (1993) 5354.

[5] H. Fritzsch, Phys. Lett. 73B (1978) 317; Nucl. Phys. 115B (1979) 189.

[6] G.C. Branco and J.I. Silva-Marcos, Phys. Lett. B 331 (1994) 390.

[7] G. Altarelli and F. Feruglio, Phys. Lett. B 439 (1998) 112, hepph/9807353; G. Altarelli and F. Feruglio, hep-ph/9812475; Z. Berezhiani and A. Rossi, hep-ph/9811447; K. Hagiwara and N. Okamura, hepph/9811495.

[8] J. K. Elwood, N. Irges, and P. Ramond, Phys. Rev. Lett. 81 (1998) 5064, hep-ph/9807228 and references contained therein.

[9] C.H. Albright and S.M. Barr, in preparation.

[10] M. Gell-Mann, P. Ramond, and R. Slansky, Report No. CALT-68-709: Supergravity (North Holland, Amsterdam, 1979); T. Yanagida, in Proceedings of the Workshop on Unified Theory and Baryon Number of the Universe, KEK, Japan, 1979 (unpublished).

[11] L. Wolfenstein, Phys. Rev. Lett. 51 (1983) 1945.

[12] Table of Particle Properties, Particle Data Group, 1998. 


\section{Figure Captions}

Fig. 1: The diagram that generates the " $\epsilon$ " entries of the quark and lepton mass matrices.

Fig. 2: The diagram that generates the " $\sigma$ " entries of the mass matrices $L$ and $D$.

Fig. 3: The diagrams that produce small masses for the first-family quarks and leptons. The family index $j$ on the spinor field takes the values 2 or 3 , giving, respectively, the $\delta$ and $\delta^{\prime}$ terms of the mass matrices. Different $S O(10)$ vector Higgs, denoted with superscript $j$, are exchanged in the two cases.

Fig. 4 A comparison of the model with experiment for the Wolfenstein parameters $(\rho, \eta)$. The axes are $\rho$ and $\eta$ multiplied by the central value of $\left|s_{12} V_{c b}\right|$. The central values allowed by the model lie on the bold dashed circular arc, cf. Eq. (6). The constraints following from $\left|V_{u b}\right|, B$-mixing and $\epsilon$ extractions from experimental data are shown in the lightly shaded regions. The experimentally allowed region is indicated by the heavily shaded central region. A typical unitarity triangle allowed by both data and the model is shown. 


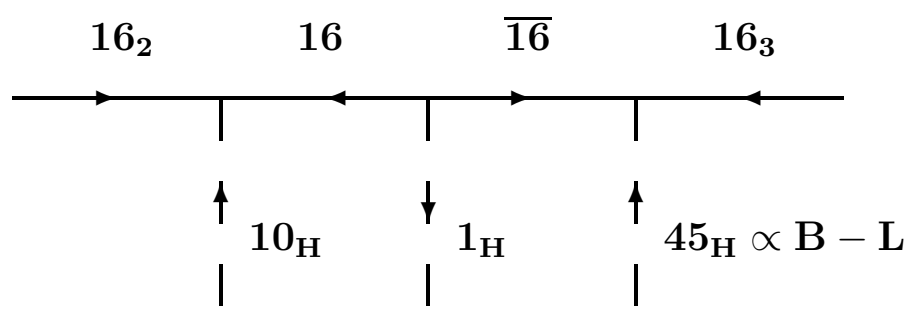

Fig. 1

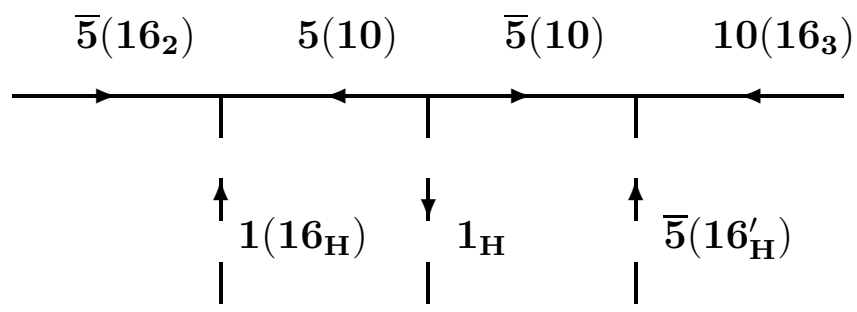

Fig. 2

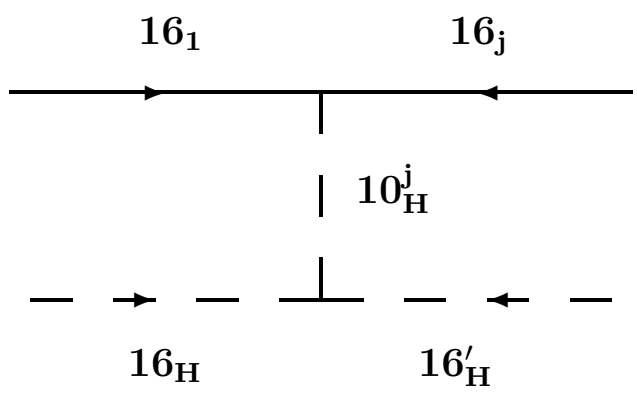

Fig. 3 


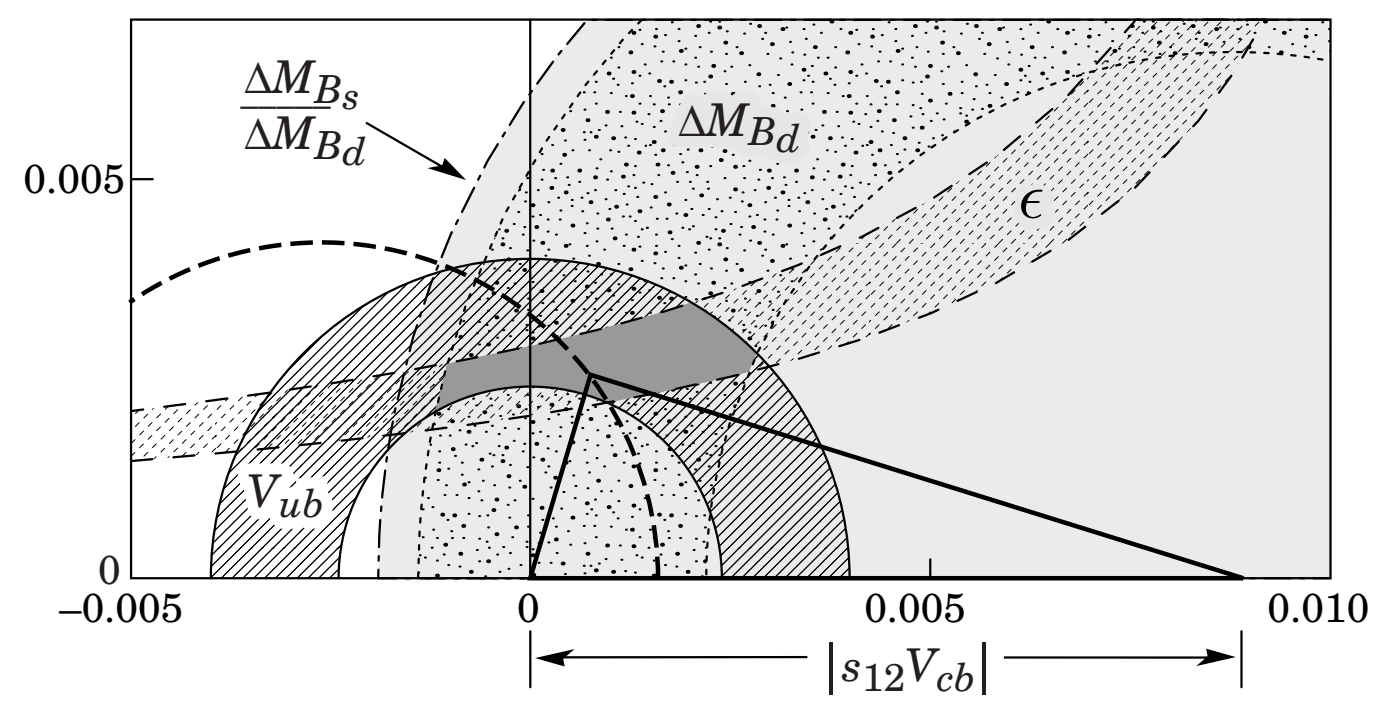

Fig. 4 